\title{
COMMUNICATIONS DEPENDABILITY EVOLUTION
}

\author{
Between Convergence and Competition
}

Michele Morganti

Siemens Mobile Communications, Italy_michele.morganti@siemens.com

\begin{abstract}
The high dependability standards that once characterized telecommunications networks were largely the consequence of the monopolistic regimes under which they were built and operated. With the progressive liberalization and deregulation of telecommunication markets this situation has dramatically changed and dependability, once a self imposed obligation, has now become a product differentiator that needs to be justified in terms of higher value for the customers and lower costs of ownership for the network operator.
\end{abstract}

Key words: Communications, Dependability, Convergence, Liberalization, Deregulation

\section{ONCE UPON A TIME ...}

Telecommunications were synonymous of public telephone networks, were almost anywhere a state monopoly, and their dependability was almost legendary.

The association of these three elements (telephony, monopoly and dependability) is definitely not accidental.

The fact, for example, that telephony was the only service officially to be delivered by the network meant a very specific and consistent set of Quality of Service (QoS) requirements and, as a direct consequence, a rather limited variance in network architectures and technological solutions.

Monopoly, on its side, meant that in each country there was only one network infrastructure, only one network operator and, obviously, only a few accredited suppliers, all of whom were willingly collaborating (among 
themselves and with operators) to defend their shares of an essentially closed and captive market.

Monopoly also meant only one source for services that were essential for the economical development of the country and for the welfare of its citizens and, at the same time, no real need to match offer and demand, with social costs usually charged to the high (and supposedly wealthy) consumers.

In this situation, dependability was perceived almost as an obligation to the country, in exchange for the privilege of monopoly, and the very high standards set for it were usually self imposed.

\section{A LONG WAY FORWARD}

Over time, many different and important events have contributed to determine an almost complete upturn of the above situation, some of them technological, some cultural, and some directly related to evolving market conditions and new regulations.

Historically, the first of these changes was the digitalization of telephone networks, and the most recent one the diffusion of public wireless access.

\subsection{Digitalization}

Digitalization initiated almost 40 years ago, and was initially aimed at improving the efficiency of long haul transmission, at the time one of the most expensive components of telecommunications networks. From there it quickly extended to all other network elements (although even today it cannot be said complete, as many analog local loops still exist in most countries). Digitalization contributed to the evolution of dependability in several different ways.

First of all it forced engineers to transpose requirements and objectives originally set for electromechanical equipment managing single circuits to computer controlled systems managing several thousand circuits at one time. Furthermore, computers and electronics in general were at the time fairly new, unreliable, and also very expensive objects, that needed to be used with parsimony. The challenge was in itself so big that the result was a large variety of architectural solutions, all of them embedding sophisticated fault tolerance techniques and various levels of hardware redundancy.

Another major consequence of digitalization was that by changing voice into a stream of bits it made it undistinguishable from data as well as from any other digitalized content. This fact did not produce an immediate effect on the architecture of telecommunications networks, but opened the way to another important historical period that went under the name of convergence. 


\subsection{Convergence}

Convergence is in itself a fuzzy concept and, therefore, also a term that is easily abused and misused. Whether applied to technology, infrastructures, services or even market segments, it has often been invoked to assert one thing and the contrary at the same time.

Its first appearance in telecommunications referred to the theoretical possibility that, through digitalization, the same (first circuit, then packet based) infrastructure could be used to deliver all services and contents.

Eventually, everybody had to acknowledge that requirements were in most cases so different and conflicting that a single network could never satisfy them all, both technically and economically. Hence, the most evident effect of convergence on communications was a sort of creative divergence that fostered many new technologies, new network architectures, and even entirely new communication paradigms, like the Internet.

Eventually, some solutions proved to be intrinsically more pervasive and general purpose than others. However, convergence days demonstrated that there is always value in diversity, that one size does not always fit all, and that communications are there to satisfy people needs and not to create them.

\subsection{The Internet}

Talking dependability, the main lesson that came from the Internet is that reliable communications can be built on top of relatively unreliable network elements. Until then it had been common belief in the telecommunications community that this could only be done by purpose design, and that circuit based networks were intrinsically more dependable than packet ones just because their behavior was easier to predict and to engineer.

The Internet also proved that distributed control can be as (or even more) effective than centralized one, and that best results are achieved when there is intelligence both inside and outside of the network. At the time, the typical architecture of public telecommunications networks was strictly hierarchical, with most of the intelligence concentrated in the network core. Terminals were generally assumed to be dumb and, therefore, unable to contribute to the overall performance of the network or to dynamically adapt its status.

Finally, the Internet community showed to the telecommunications world that there could be forms of governance much simpler, faster and effective than those provided by official standardization bodies, and that, especially in a fast changing world, it was unwise to set standards as broad, detailed and rigid as it had been the case until then. Although it took quite some time for the Internet lessons to be fully understood and accepted, success stories like GSM, MPEG and WiFi could never have happened without them. 


\subsection{Privatization, Liberalization and Deregulation}

Liberalization of telecommunications initiated almost a decade ago and quickly became a global phenomenon.

By introducing competition and by forcing formerly public operators to justify all of their operational costs to private investors, liberalization also obliged them to reassess all of their business practices, including those relevant to the realization of network infrastructures. Furthermore, many regulators decided to relax a number of previous obligations (also in term of minimum QoS requirements) in order to reduce entry barriers for the new operators.

In this context, the previous high network dependability standards, which in most cases implied massive hardware redundancy without clear evidence of a corresponding market benefit, became an easy target for CAPEX cutting measures.

The new cell based ATM switches and cross connects (which were at the time under development by a number of leading manufacturers worldwide) were the first and most illustrious victims of the new era. Their extremely demanding specifications had in fact become too expensive for those same operators who had originally imposed them.

This also caused a definitive rupture in the previously intimate relationships between operators and manufacturers. From there on operators took a strictly opportunistic behavior (take no commitment and buy what is available when you really need it), while manufacturers were obliged to assume full risk and responsibility for all new product developments.

\subsection{Mobile Communications}

The QoS of mobile communications is intrinsically lower than that of fixed ones. The radio resource is scarce and there are numerous constraints, interferences and physical obstacles that further reduce its efficiency of use. Nevertheless, the exceptional market response they got demonstrated that people are willing to accept all of these limitations and still pay a premium for the several other advantages they get. In short, they represent the outstanding proof that dependability and QoS are, to a large extent, options that need, as everything else, to be justified in terms of additional market share and value recognition.

They also demonstrated that steady state availability (until then the main design goal of telecommunications engineers) is only one of the many components of network dependability that need to be taken care of, and not necessarily the most important one. As a matter of fact, unavailability in mobile networks is more frequently due to poor coverage or to access congestion than to equipment failures. 
On the other hand, mobile networks require considerable intelligence in the perifery, and this implies that their operational costs are much more influenced by equipment reliability than those of fixed ones. Terminals too are orders of magnitude more complicated, and still they must provide continuous fault free operation for several years.

Mobile handsets also include a number of specific dependability features that allow them to deal with the many adverse situations that they can meet in a hostile mobile environment. For example, most of them can deal with multiple networks and network technologies at the same time and then choose the best one (e.g., 3G terminals automatically switch to $2 \mathrm{G}$ service if signal quality drops below a certain level). Further, they dynamically adapt their transmit power and rate to actual transmission conditions.

Other dependability aspects that require special attention in designing mobile networks are data security and integrity, customer localization and tracking, and, consequently, customer data privacy. It is common knowledge that, in recent years, several criminals have been captured thanks to their incautious use of mobile phones, and with the introduction of A-GPS, location accuracy will further improve to within just a few meters.

\subsection{Wireless Ubiquity}

Public WiFi Hot-Spots made their appearance a couple of years ago, and immediately caught public attention.

Conceived for private usage in confined indoor environments, WiFi is a relatively unreliable and rather insecure technology that operates in a very crowded and noisy portion of unlicensed spectrum: totally unsuitable, one would say, for public usage and definitely unable, in this context, to guarantee any significant level of QoS. That it was immediately regarded as a concrete threat to mobile communications, and more specifically to $3 \mathrm{G}$, is a clear indication of the fact that even operators had no clear understanding of how customers would react to the new offer, however unreliable.

Eventually public Hot-Spots disappointed the unrealistic expectations of some financial analysts. However, this was due more to coverage limitations than to their lack of QoS guarantees, including dependability related ones.

Technicians are now working to complement WiFi through WiMAX. Conceived for outdoor usage, WiMAX is definitely more robust that $\mathrm{WiFi}$, will be operated in both licensed and unlicensed spectrum and will offer $70 \mathrm{Mb} / \mathrm{s}$ access over cells that are the same size of mobile ones. In the new versions of the standard, WiMAX could also support some limited mobility.

Although WiMAX is IP native (and therefore intrinsically unable to guarantee certain QoS classes) wireline operators (especially alternative ones) see it as a perfectly viable alternative to xDSL over copper pairs while 
mobile operators are seriously considering it as an inexpensive solution for their back-hauling networks.

At the same time, silicon and terminal manufacturers are committed to embed WiFi and WiMAX capabilities directly into Lap-Tops, PDA's and even handsets. Further, almost everybody already agrees that $4 \mathrm{G}$ will not be a single new network but many different ones, each with its own specificity, to be used in alternative or in combination depending on instantaneous availability and needs.

\section{IN CONCLUSION}

Some ten years ago communications dependability had become a sort of routine work: on the one side there was a set of networks elements with precise functionalities and requirements; on the other, a matching set of design alternatives that could be used to implement them. Both sets had been consolidated through almost thirty years of experience, and it was common understanding that there was little more left to invent and no real motivation to justify the effort.

Liberalization and mobility completely upturned this situation and forced network operators and their suppliers to completely reassess, in terms of real market value, their traditional design practices and architectural choices. They had to recognize, for example, that IP backbones, although less predictable than SDH ones, could indeed provide, through over-sizing, a still adequate and much less expensive alternative. Similarly, in most cases a temporary roaming agreement with a competitor could prove more effective and less expensive than extensive redundancy to ensure continuity of service in case of mobile access equipment failures.

In this fast evolving business context, manufacturers are obliged to make their own choices and to take their own risks. Operators, on their part, need to justify all of their investments in terms of additional market opportunities and higher margins.

Dependability too, once simply a must, has become an option that needs to be tuned, on a case by case basis, to actual needs and opportunities. Terms like QoS and redundancy scalability have become commonplace in product descriptions. At the same time, however, the scope of dependability has much broadened to include once disregarded aspects like security and safety.

Looking to the future, the main challenge is no more how to build highly dependable networks, but how to get the best out of many unreliable ones. 